\title{
Unsteady turbulence cascades
}

\author{
Susumu Goto \\ Graduate School of Engineering Science, Osaka University, \\ 1-3 Machikaneyama, Toyonaka, Osaka, 560-8531 Japan \\ J.C. Vassilicos \\ Department of Aeronautics, Imperial College London, London, United Kingdom
}

\begin{abstract}
We have run a total of 311 Direct Numerical Simulations (DNS) of decaying three-dimensional Navier-Stokes turbulence in a periodic box with values of the Taylor length-based Reynolds number up to about 300 and an energy spectrum with a wide wavenumber range of close to $-5 / 3$ power-law dependence at the higher Reynolds numbers. On the basis of these runs we have found a critical time when (i) the rate of change of the square of the integral length-scale turns from increasing to decreasing, (ii) the ratio of interscale energy flux to high-pass filtered turbulence dissipation changes from decreasing to very slowly increasing in the inertial range, (iii) the signature of large-scale coherent structures disappears in the energy spectrum and (iv) the scaling of the turbulence dissipation changes from the one recently discovered in DNS of forced unsteady turbulence and in wind tunnnel experiments of turbulent wakes and grid-generated turbulence to the classical scaling proposed by G.I. Taylor in 1935 and A.N. Kolmogorov in 1941. Even though the customary theoretical basis for this Taylor-Kolmogorov scaling is a statistically stationary cascade where large scale energy flux balances dissipation, this is not the case thoughout the entire time-range of integration in all our DNS runs. The recently discovered dissipation scaling can be reformulated physically as a situation where the dissipation rates of the small and the large scales evolve together. We advance two hypotheses which may form the basis of a theoretical approach to unsteady turbulence cascades in the presence of large-scale coherent structures.
\end{abstract}

\section{INTRODUCTION}

Non-linear dynamics present a major challenge to much of current physics. In high Reynolds number turbulent flows non-linear dynamics generate an energy cascade which determines the dissipation properties of the turbulence. The turbulence dissipation rate is key for determining the number of degrees of freedom, drag, turbulence decay, turbulence diffusion, entrainment and mean flow profiles in various turbulent flows (see $[20,25])$. An understanding of the non-linear energy cascade in NavierStokes turbulence can be expected to have an even wider impact if it can serve as a paradigm or reference case for energy cascades in many other physical systems governed by non-linear field equations.

In many turbulent flows, such as various turbulent wakes and jets and also turbulence generated by various types of grids, the turbulence starts by building up along the downstream direction and then, when the turbulence production becomes insufficient, carries on decaying further downstream. Recent wind tunnel experiments have revealed a dissipation law in the decay regions of various turbulent wakes and various grid-generated turbulent flows which appears to belong to some universality class but is at odds with Kolmogorov's equilibrium cascade (see $[4,15,25])$. Kolmogorov's equilibrium cascade is the only type of turbulence cascade for which an accepted theory exists; it has been a cornerstone or at least a term of reference for nearly all predictive and modelling approaches and theories of turbulence since the 1940s. In the remainder of this paper we use the terms "statistically stationary cascade" and "Kolmogorov stationar- ity" when refering to Kolmogorov's equilibrium cascade to avoid confusions which can arise from the various uses of the word "equilibrium" in physics and mechanics in general.

The dissipation law implied by Kolmogorov's statistically stationary cascade is $\epsilon=C_{\epsilon} K^{3 / 2} / L$ where $K$ is the turbulent kinetic energy per unit mass, $\epsilon$ is the rate of dissipation of turbulent kinetic energy per unit mass, $L$ is an integral length-scale and $C_{\epsilon}$ is a dimensionless constant. The new dissipation law mentioned above is such that $C_{\epsilon}$ is not constant but proportional to the square root of a global/inlet/inital Reynolds number divided by the local Taylor length-based Reynolds number $R_{\lambda}$ (see $[4,15,25])$. Recently we have confirmed and in fact extended the universality of this new dissipation law $[8,9]$ by showing that it is also present in Direct Numerical Simulations (DNS) of both forced and decaying incompressible Navier-Stokes turbulence with periodic boundary conditions in all three directions. In the forced case it is imperative that the resulting turbulence should be unsteady over significant time scales for this new dissipation law to hold (see $[8,25])$. Steady turbulence is an exceptional case of turbulence where the Kolmogorov statistically stationary cascade rules and the dissipation scaling is the well known classical scaling (see $[5,8,25]$ ).

Wind tunnel experiments of grid-generated turbulence have also shown, however, that there can exist a far downstream region $[10,23,25]$, downstream of the decay region described two paragraphs above, where the dissipation law appears classical. There is no evidence for such change of behaviour in DNS of forced unsteady turbulence $[8,9]$, but in this paper we report that there ac- 
tually is something similar in DNS of decaying spatially periodic turbulence.

This paper reports on DNS of freely decaying periodic turbulence and is organised as follows. In section II we show that the new dissipation law holds in a first period of decay which lasts a few turnover times and that it is then followed by a second period where the relation between dissipation rate and Reynolds number is radically different from the new dissipation law.

In section III we correlate this relatively abrupt change in dissipation behaviour to an equally abrupt change in the evolving unsteadiness of the small-scale turbulence. In section IV we discuss an existing non-Kolmogorov theory of decaying turbulence and confront it with our DNS data. Then, in section $\mathrm{V}$, we transform the new dissipation law into an equivalent statement which is easier to interpret physically and in section VI we show how the hypotheses of the longest standing non-Kolmogorov theory of decaying turbulence can be reduced and weakened to avoid disagreement with our DNS data and at the same time obtain the new dissipation law. In section VII we derive the consequences of our new approach for the interscale energy flux and in section VIII we introduce the concept of unsteady balance in an attempt to account for the properties of the second period of decay. In section IX we conclude with a summary and short discussion of our results.

\section{TURBULENCE DISSIPATION SCALING}

Our DNS of decaying turbulence are identical to the DNS of decaying turbulence of [8]. We numerically integrate the Navier-Stokes equations with periodic boundary conditions (periodic domain of box size $2 \pi$ ) in three orthogonal direction using the fourth-order RungeKutta-Gill scheme. The spatial derivatives are evaluated by Fourier spectral method where aliasing errors are removed by the phase-shift technique. Like [8] we first run a forced DNS with their exact same forcing $\mathbf{f}$ on the right hand side of the Navier-Stokes equation, i.e. $\mathbf{f}=\left(-\sin \left(k_{f} x\right) \cos \left(k_{f} y\right), \cos \left(k_{f} x\right) \sin \left(k_{f} y\right), 0\right)$ with $k_{f}=4$ and, also like them, we turn off the forcing at time $t_{0}$ when the dissipation has reached a maximum. We subsequently let the turbulence decay. (We have also repeated this procedure in some cases with a random white-in-time forcing and found similar results as the ones reported in this paper.)

The initial condition consists of small disturbances over all wavenumbers $k$ with energy spectrum proportional to $k^{-1}$ and random phases. The initial condition's energy (per unit mass) is $O\left(10^{-4}\right)$, while the energy at $t_{0}$, when the forcing is switched off, is $O(1)$. We run many simulations with many such initial conditions produced by different sets of random phases. The results we present in this paper are ensemble averages over these runs. We run a number $N_{R}$ of simulations per simulation size $N^{3}$;
$N_{R}=100$ for $N=128,256$, and $512, N_{R}=10$ for $N=1024$ and $N_{R}=1$ for $N=2048$. The spatial resolution $k_{\max } \eta$ (where $k_{\max }$ is the largest resolved wavenumber and $\eta$ is the Kolmogorov microscale) is slightly above 1 at $t_{0}$ for all these simulations. Higher values of $N$ correspond to higher Reynolds numbers and $k_{\max } \eta$ increases throughout the decay.

The integral length-scale is calculated from

$$
L(t)=\frac{3 \pi}{4} \int_{0}^{\infty} k^{-1} E(k, t) d k / K(t)
$$

(where $E(k, t)$ is the energy spectrum and $K(t)$ is the turbulent kinetic energy per unit mass) and varies somewhat from realisation to realisation but is always within $\pm 20 \%$ of the ensemble-averaged integral length scale $\bar{L}(t)$ which we plot in Fig. 1. In this figure we plot both $\bar{L}$ and $\frac{d}{d t} \bar{L}^{2}$ as functions of the number of turnover times

$$
\widehat{t} \equiv \int_{t_{0}}^{t} \frac{\overline{u^{\prime}}}{\bar{L}} d t
$$

where $\frac{3}{2} u^{\prime 2}(t)=K(t)$ and $\overline{u^{\prime}}(t)$ is ensemble-averaged. Our definition of $\widehat{t}$ assumes that each one of our ensembles of $N_{R}$ simulations is statistically equivalent to a single very large domain simulation which would contain a number of integral scale regions equal to the sum of integral length scale regions in each realisation of the ensemble. There is no reason to expect our results to be so sensitive to subtle differences in the statistical definition of $\widehat{t}$.

Looking at $\frac{d}{d t} \bar{L}^{2}$ one can make two observations. Firstly, the sudden removal at time $t=t_{0}$ (i.e. $\widehat{t}=0$ ) of the force $\mathbf{f}$ removes a long range correlating agent and the integral scale starts to decrease till a time $t_{g}$ when it starts growing again because of the natural growth of the largest eddies of freely decaying turbulence. Secondly, after $t_{g}$ there is a second time $t_{c}$ when $\frac{d}{d t} \bar{L}^{2}$ reaches a maximum. Note that $\bar{L}(t)$ is smaller than a tenth of the box size $2 \pi$ from $\widehat{t}=0$ to $\widehat{t}=4$ for our three simulation ensembles with the highest Reynolds numbers (see [21] where it is shown that the integral length-scale is not significantly affected by the domain size if it is a tenth of that size or less) and that the range $\widehat{t} \leq 4$ includes the time $t_{c}$. In fact there is about one turnover time from $t=t_{c}$ to $\widehat{t}=4$. Note also that there are typically two or more turnover times from $t_{g}$ to $t_{c}$.

The ensemble-averaged Reynolds numbers $\overline{R_{\lambda}}(t)$ are plotted in Fig. 2; $R_{\lambda}(t) \equiv u^{\prime}(t) \lambda(t) / \nu$, where $\lambda$ is the Taylor microscale and $\nu$ is the kinematic viscosity. One can also define a Reynolds number based on the integral length scale, i.e. $u^{\prime}(t) L(t) / \nu$, and at time $t=t_{0}$ this Reynolds number is $R_{0} \equiv u^{\prime}\left(t_{0}\right) L\left(t_{0}\right) / \nu$. In some instances we may refer to the ensemble-averaged Reynolds number $\overline{R_{0}}$. Note that the highest Reynolds numbers achieved in our DNS of freely decaying turbulence are the highest ever for integration times as long, ratios of box size to integral length scale as large and small-scale 

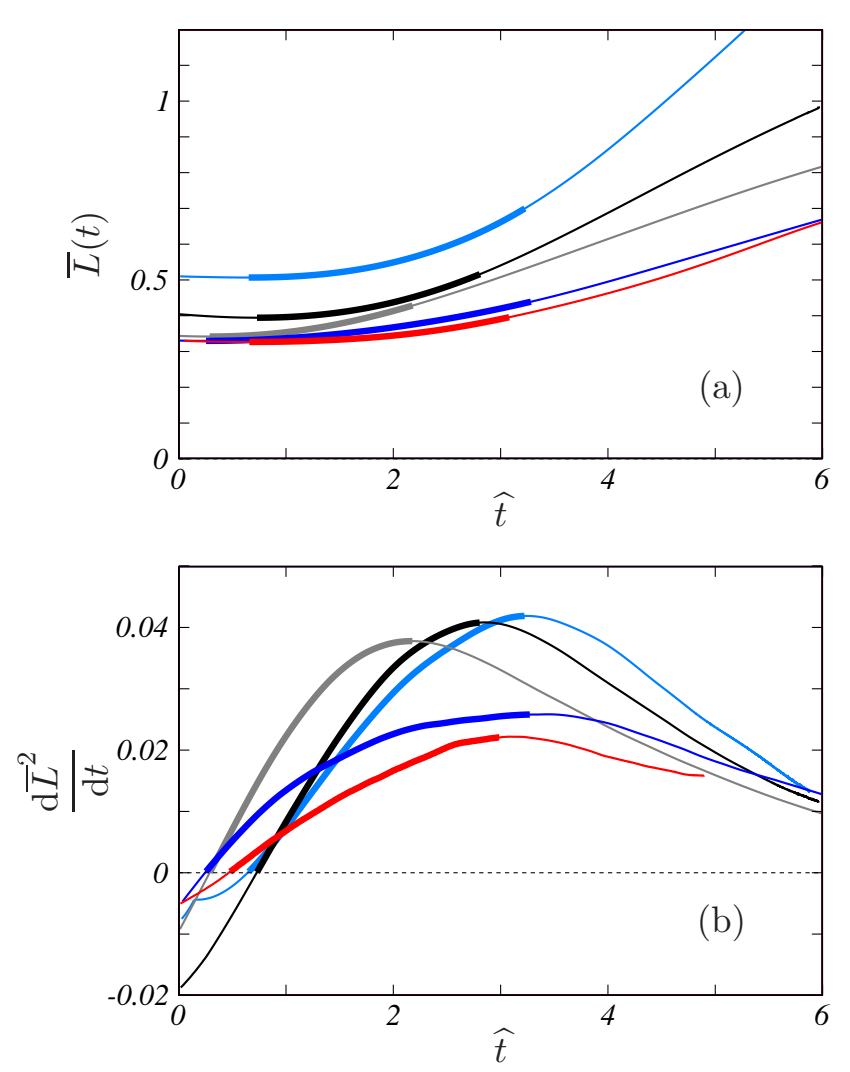

FIG. 1. Average integral scale $\bar{L}$ (a) and $\frac{d \bar{L}^{2}}{d t}$ (b) versus number of turnover times $\widehat{t}$ for different simulation ensembles. Red $N=2048$; blue $N=1024$; grey $N=512$; black $N=256$; light blue $N=128$. See Fig. 2 for how this colour coding corresponds to different Reynolds numbers. The thick part of the lines marks the range $t_{g} \leq t \leq t_{c}$ where $t_{g}$ is the time when $\frac{d \bar{L}^{2}}{d t}$ starts being positive and $t_{c}$ is the time when $\frac{d \bar{L}^{2}}{d t}$ stops growing.

resolutions as good as here. Higher values of $R_{\lambda}$ have been achieved with DNS of forced periodic turbulence where the integral scale is comparable to the box size (see e.g. [27]).

Fig. 3 shows how the dissipation coefficient $C_{\epsilon}$ (defined by $\epsilon=C_{\epsilon} u^{\prime 3} / L=C_{\epsilon}(2 K / 3)^{3 / 2} / L$ where $\epsilon(t)$ is the turbulence dissipation per unit mass) starts off, once the forcing has been removed, as an increasing function of time till it quite abruptly turns into an approximate constant. This change of trend is clear in the insert of Fig. $3(\mathrm{a})$ where values of $C_{\epsilon}$ are plotted versus time for 10 different realisations of the $N=512$ simulation ensemble and in the main Fig. 3(a) where the ensemble-averaged $\overline{C_{\epsilon}}$ is plotted for each one of our five simulation ensembles. Incidentally, it is remarkable how our slightly differing initial conditions at time $t=0$ (several eddy turnover times prior to $t=t_{0}$ ) can lead to up to $20 \%$ differences in the long time values of $C_{\epsilon}$, an observation which is about the same for all the Reynolds numbers we are trying here. It is this observation and similar ones for the integral length-scale by us and by [21] which have forced

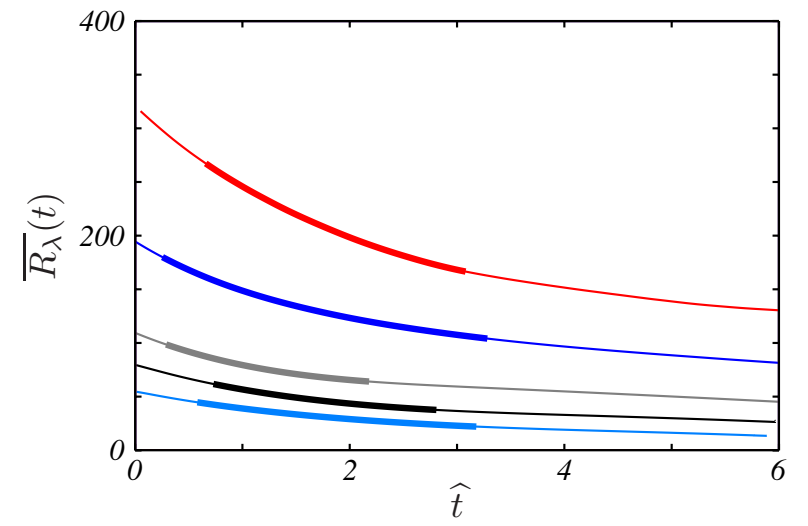

FIG. 2. $\overline{R_{\lambda}}(t)$ versus $\widehat{t}$ for the five simulation ensembles. Red $N=2048$; blue $N=1024$; grey $N=512$; black $N=256$; light blue $N=128$. The thick parts of the lines mark the range $t_{g} \leq t \leq t_{c}$.

us to present our results in terms of ensemble averages. This means, in particular, that our results for $N=2048$ (where $N_{R}=1$ ) are meaningful only qualitatively.

The change of trend observed in Fig. 3 is effectively the same as the one observed in wind tunnel experiments of grid-generated turbulence (compare Fig. 3 with Fig. 4 in [25] where this change of trend is apparent in wind tunnel experiments) where streamwise distance corresponds to time in our DNS. In Fig.4 we plot $\overline{C_{\epsilon} / \sqrt{R_{0}}}$ versus $\overline{R_{\lambda}}$ which we have actually found to be nearly identical to $\overline{C_{\epsilon}} / \sqrt{\overline{R_{0}}}$ versus $\overline{R_{\lambda}}$. This figure shows that our data collapse is in some agreement with the new dissipation law for the higher Reynolds numbers of each simulation ensemble (see also uncollapsed data in the figure's insert), i.e. $\overline{C_{\epsilon} / \sqrt{R_{0}}} \sim 1 / \overline{R_{\lambda}}$ or $\overline{C_{\epsilon}} \sim \sqrt{\overline{R_{0}}} / \overline{R_{\lambda}}[4,8,9,15,25]$. It is also clear from the thick parts of the curves in Fig. 3 and Fig. 4 that the time $t_{c}$ when the rate of growth of the square integral length suddently stops increasing and starts decreasing is also the time when the timedependence of the dissipation coefficient $C_{\epsilon}$ qualitatively changes trends. (Even though this two-stage behaviour is clear, $C_{\epsilon}$ is not perfectly constant at times $t>t_{c}$, particularly for the lower Reynolds number cases.)

\section{UNSTEADINESS}

High Reynolds number turbulence dissipation is the result of a turbulence cascade. To study the turbulence cascade in homogeneous or periodic turbulence one often starts from the scale-by-scale energy balance

$$
\frac{\partial K^{>}(k, t)}{\partial t}=\Pi(k, t)-\epsilon^{>}(k, t)
$$



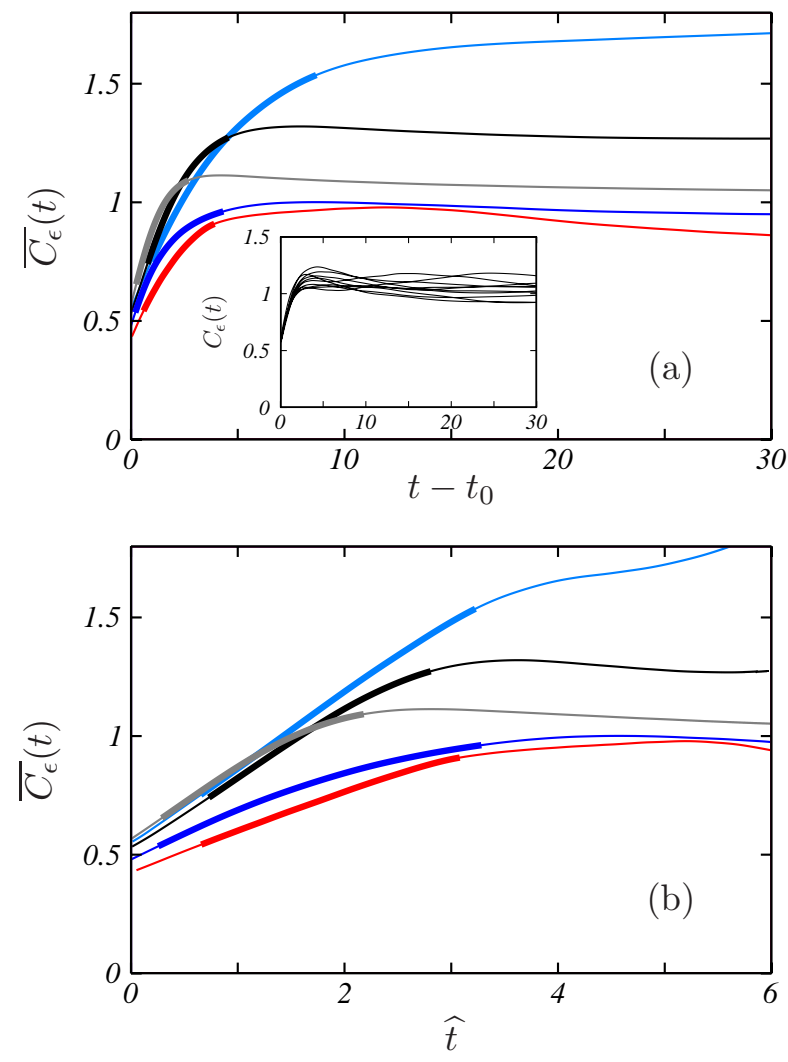

FIG. 3. $\overline{C_{\epsilon}}$ versus (a) time $t-t_{0}$ and (b) number of turnover times $\widehat{t}$ for five simulation ensembles. See Fig. 2 for the colour coding corresponding to different Reynolds numbers. The thick part of the lines marks the range $t_{g} \leq t \leq t_{c}$. The insert in (a) is a plot of $C_{\epsilon}$ versus time for 10 different realisations of the $N=512$ simulation ensemble (grey curves in the main plots).

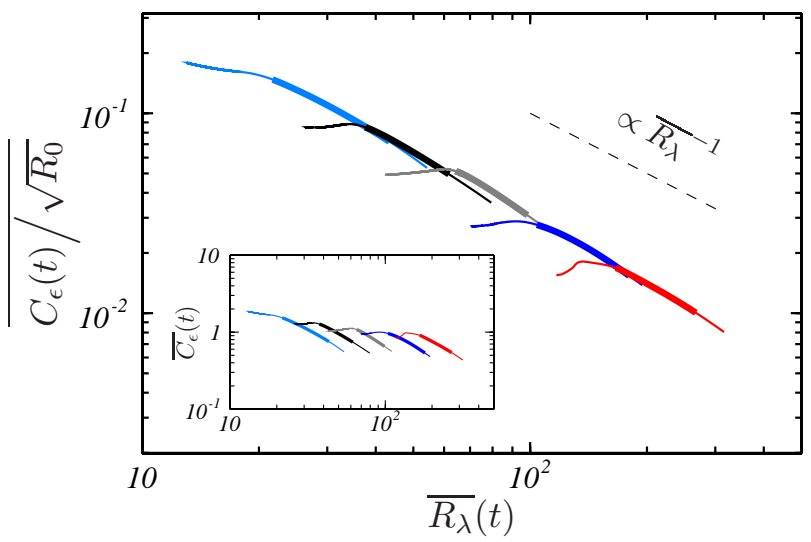

FIG. 4. Log-log plot of $\overline{C_{\epsilon}(t) / \sqrt{R_{0}}}$ versus $\overline{R_{\lambda}}(t)$. The dotted line in the plot represents $\bar{R}_{\lambda}-1$. See Fig. 2 for the colour coding corresponding to different Reynolds numbers. The insert is a log-log plot of $\overline{C_{\epsilon}}(t)$ versus $\overline{R_{\lambda}}(t)$. The thick part of the lines marks the range $t_{g} \leq t \leq t_{c}$.

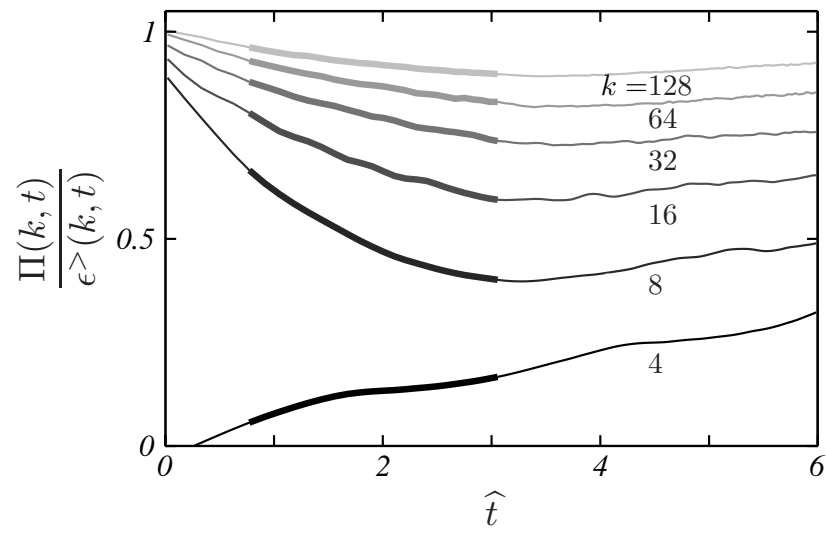

FIG. 5. Interscale energy flux to wavenumbers larger than $k$ divided by the turbulence dissipation in wavenumbers larger than $k$. This ratio's departure from 1 is a measure of how unsteady the cascade is at wavenumber $k .2048^{3}$ run; curves from black to grey and from the lower to the upper parts of the plot correspond to $k=4,8,16,32,64,128\left(k_{f}=4\right)$.

where we use the same notation as [9] defined in terms of the energy spectrum $E(k, t)$ :

$$
K^{>}(k, t) \equiv \int_{k}^{\infty} E(k, t) d k
$$

and

$$
\epsilon^{>}(k, t) \equiv 2 \nu \int_{k}^{\infty} k^{2} E(k, t) d k
$$

are, respectively, the turbulent kinetic energy and the turbulence dissipation in wavenumbers larger than $k$ and $\Pi(k, t)$ is the interscale flux of turbulent kinetic energy from wavenumbers smaller to wavenumbers larger than $k$ (we omit "per unit mass" in all three cases for brevity). Kolmogorov stationarity is a situation where

$$
\left|\frac{\partial K^{>}(k, t)}{\partial t}\right| \ll \epsilon^{>}(k, t)
$$

and therefore

$$
\Pi(k, t) \approx \epsilon^{>}(k, t)
$$

at wavenumbers $k$ smaller than wavenumbers where viscous effects dominate.

The forcing is at a small wavenumber of the DNS, i.e. a length-scale larger than $L\left(t_{0}\right)$ in every realisation of our simulation ensembles. Before cutting off the forcing, which in our case is at wavenumber $k_{f}=4<2 \pi / L$, one may expect small values of $\Pi(k, t)$, i.e. close to 0 , for $k \leq k_{f}$.

At wavenumbers $k>k_{f}$, while the forcing might have been setting a tendency towards a Kolmogorov statistical stationarity $\Pi(k, t) \approx \epsilon^{>}(k, t)$ at the higher wavenumbers, the removal of the forcing just leaves us with (1). The cascade time-lag observation of [3] and [9] implies that $\Pi(k, t)$ will react to the absence of large-scale powerinput well before $\epsilon^{>}(k, t)$ has had time to react to it. 
$\Pi(k, t)$ will therefore accelerate its decline before $\epsilon^{>}(k, t)$ does so too. Hence, we must expect $\Pi(k, t) / \epsilon^{>}(k, t)$ to decrease with time for all $k>k_{f}$. This implies that $\frac{\partial K^{>}(k, t)}{\partial t}$ increases in magnitude with time for these wavenumbers. There must therefore be a tendency for increased unsteadiness in the wavenumber range $k>k_{f}$ after removal of the forcing, in other words a departure from Kolmogorov stationarity which increases with time.

These expectations are confirmed up to a point by our DNS of periodic turbulence decay. Fig. 5 is a plot of $\Pi(k, t) / \epsilon^{>}(k, t)$ as a function of number of turnover times $\widehat{t}$ for various wavenumbers. $\Pi(k, t) / \epsilon^{>}(k, t)$ decreases sharply for all wavenumbers larger than $k_{f}=4$ till the same time $t_{c}$, irrespective of wavenumber, when $\frac{d}{d t} \bar{L}^{2}$ and $\overline{C_{\epsilon}}$ change trends. At times larger than $t_{c}$, $\Pi(k, t) / \epsilon^{>}(k, t)$ exhibits a slow growth. We can expect a long time $t_{\nu}(k)$ to exist when $\epsilon^{>}(k, t)$ is very much smaller than $\epsilon$ and $K^{>}(k, t)$ very closely vanishes. At such long times $\Pi(k, t) / \epsilon^{>}(k, t)$ is dominated by direct viscous effects. Note, when looking at Fig. 5, that $k=128$ corresponds to a wavelength of about $10 \eta$ at $\hat{t}=6$ for the $N=2048$ simulation.

Our preliminary considerations at the start of this section prepared us for an initial decrease of $\Pi(k, t) / \epsilon^{>}(k, t)$ at large enough wavenumbers resulting from the timelag imposed by the energy cascade $[3,9]$ in an unsteady turbulence (such as decaying turbulence). But they did not prepare us for a critical time when $\Pi(k, t) / \epsilon^{>}(k, t)$ at such wavenumbers change behaviour and start growing very slowly, nor did they prepare us for this critical time to be $t_{c}$, the time when $\frac{d}{d t} \bar{L}^{2}$ changes trends and when the dissipation scaling transitions from an increasing function of time similar to $\overline{C_{\epsilon}} \sim \sqrt{\overline{R_{0}}} / \overline{R_{\lambda}}$, to a slow varying function near an approximate constant.

The time range $t_{0}<t<t_{c}$ is therefore, indeed, an increasingly non-stationary range given that $\left|\frac{\partial K^{>}}{\partial t}\right| / \epsilon^{>}$ increases with time and therefore moves, progressively, further and further away from Kolmogorov stationarity. Note that the time range $t_{c}<t<t_{\nu}(k)$ is also a nonstationary range given that $\left|\frac{\partial K^{>}}{\partial t}\right| / \epsilon^{>}$is never negligibly small in that range. In our data the turbulence is always well away from Kolmogorov stationarity. Such stationarity might be reached at very much higher Reynolds numbers and much longer times if the time $t_{s}(k)$ to reach it is smaller than $t_{\nu}(k)$ for a significant range of wavenumbers smaller than where the dissipation range starts. Simulations and experiments which can reach such very far time ranges at such extremely high Reynolds numbers seem impossible nowadays and will remain so for many years to come. This point also suggests that Kolmogorov stationarity, even if it were to exist in unsteady turbulence, would be of limited relevance.

In the next section we start with a critical examination of what we believe to be the longest standing nonKolmogorov theory of unsteady small-scale turbulence by George [6] and Barenblatt \& Gavrilov [1] and confront it with our DNS data. We then advance a few hypotheses in sections $\mathrm{V}$ and VI which are quite powerful but nevertheless weaker than the hypotheses underpinning the theory of $[1,6]$ and which can account for some aspects of freely decaying unsteady turbulence.

\section{AN EXISTING NON-KOLMOGOROV THEORY OF FREELY DECAYING TURBULENCE}

To our knowledge, there are two currently available non-Kolmogorov theories of unsteady turbulence, one by Yoshizawa [28] and one by George [6]. An approach to unsteady turbulence dissipation based on [28] is currently being proposed by [2] for forced unsteady turbulence of the type discussed in [8, 9]. Here we examine George's [6] theory of freely decaying turbulence which is similar to (if not effectively the same as) the theory of Barenblatt \& Gavrilov [1] who explained how their approach is more general than Sedov's [16, 17]. Barenblatt \& Gavrilov [1] and Sedov [16, 17] worked on the Kármán-Howarth equation whereas George [6] worked on the equivalent Lin equation. Equation (1) is the integral of the Lin equation

$$
\frac{\partial}{\partial t} E(k, t)=-\frac{\partial}{\partial k} \Pi(k, t)-2 \nu k^{2} E(k, t) .
$$

George's [6] theory of decaying homogeneous turbulence hypothesises that a single length-scale $l(t)$ exists such that, for all wavenumbers $k$,

$$
E(k, t)=K(t) l(t) f\left(k l, R_{0}\right)
$$

and

$$
\Pi(k, t)=P(t) g\left(k l, R_{0}\right)
$$

where $f$ and $g$ are dimensionless functions. The prefactor $K(t) l(t)$ in $E(k, t)=K(t) l(t) f\left(k l, R_{0}\right)$ is imposed by $K(t)=\int_{0}^{\infty} E(k, t) d k$. Given the well known expressions of $L(t)$ and $\lambda(t)$ in terms of integrals over $k$ involving the energy spectrum $E(k, t)$, it is immediate that $L(t) \sim l(t) \sim \lambda(t)$. Injections of George's self-similar forms for $E(k, t)$ and $\Pi(k, t)$ in the Lin equation leads to $\frac{d}{d t}(K l)=c_{1} K \frac{d l}{d t}, P l=c_{2} K \frac{d l}{d t}$ and $2 \nu=c_{3} \frac{d l^{2}}{d t}$ where $c_{1}, c_{2}$ and $c_{3}$ are dimensionless numbers independent of time. The functions $f$ and $g$ are related by

$$
c_{1} f+\kappa \frac{\partial}{\partial \kappa} f=-c_{2} \frac{\partial}{\partial \kappa} g-c_{3} \kappa^{2} f
$$

where $\kappa \equiv k l$.

To work out what this theory's prediction is for $\epsilon$ one can use $\epsilon=-\frac{d}{d t} K$. From $\frac{d}{d t}(K l)=c_{1} K \frac{d l}{d t}$ and $2 \nu=$ $c_{3} \frac{d l^{2}}{d t}$ it follows that

$$
\epsilon=\left(1-c_{1}\right) \frac{\nu}{c_{3}} K / l^{2} .
$$

It is clear that this theory cannot lead to Kolmogorov's cascade prediction $\epsilon \sim K^{3 / 2} / L$ (see [25] and [9] for 
an explanation of how this prediction follows from the Kolmogorov stationary cascade theory). As for the new dissipation law $C_{\epsilon} \sim \sqrt{R_{0}} / R_{\lambda}$, equivalently $\epsilon \sim$ $\sqrt{K_{0}} L_{0} K / L^{2}$, George's [6] theory leads to $\epsilon \sim K / L^{2}$, i.e. $C_{\epsilon} \sim 1 / R_{\lambda}$, by setting $l(t)=L(t)$ without loss of generality. This theory therefore captures the time dependence of $\epsilon$ but the full formula $\epsilon \sim \sqrt{K_{0}} L_{0} K / L^{2}$ can only be derived from this theory with extra hypotheses concerning $c_{1}$ and $c_{3}$. Having set $l(t)=L(t)$ one can make the reasonable hypothesis that $\frac{d l^{2}}{d t}=\frac{d L^{2}}{d t}$ is independent of viscosity thereby implying that $c_{3} \sim R_{0}^{-1}$. The new dissipation law $\epsilon \sim \sqrt{K_{0}} L_{0} K / L^{2}$ follows provided one makes the additional hypothesis that $c_{1}$ is independent of $R_{0}$.

George's [6] theory also makes a prediction about the interscale flux $\Pi(k, t)$. From $P l=c_{2} K \frac{d l}{d t}$ and $2 \nu=c_{3} \frac{d l^{2}}{d t}$ it follows that $P(t)=\nu \frac{c_{2}}{c_{3}} K / l^{2}$. From (5) it then follows that

$$
P(t)=\frac{c_{2}}{1-c_{1}} \epsilon .
$$

To conclude that $\Pi(k, t)=\epsilon G(k l)$ with $G$ independent of $R_{0}$ one requires the extra, rather ad hoc, hypothesis that $G=\frac{c_{2}}{1-c_{1}} g\left(k l, R_{0}\right)$ should be independent of $R_{0}$ (at least over a particular targeted range of wavenumbers).

It is interesting that this theory can lead to

$$
\Pi(k, t)=\epsilon G(k l)
$$

which is partly similar to Kolmogorov's stationary cascade prediction $\Pi(k, t) \approx \epsilon$ in the inertial wavenumber range.

George's [6] theory neither predicts nor assumes the critical time $t_{c}$ in Fig. 1 which also appears as the critical time for a qualitative change of trends in Fig. 3, Fig. 4 and Fig. 5. Another discrepancy between George's [6] theory and our DNS is that the theory does not distinguish between wavenumbers smaller or larger than $2 \pi / L$ whereas Fig. 5 suggests that the time-dependence of $\Pi(k, t) / \epsilon^{>}(k, t)$ in our DNS is very different for such different wavenumbers in the time range $t_{0}<t<t_{c}$.

In the following sections we modify this theory in a way which respects the qualitative difference between $k \lesssim$ $2 \pi / L$ and $k \gtrsim 2 \pi / L$ yet preserves the prediction $\epsilon \sim$ $\sqrt{K_{0}} L_{0} K / L^{2}$ and replaces (6) with a relation which can account for the increasing unsteadiness in Fig. 5. In so doing we replace the hypotheses of the theory with fewer and weaker ones, some of which may also be physically more tangible.

\section{A PHYSICAL INTERPRETATION OF THE DISSIPATION SCALING $\epsilon(t) \sim K(t) / L^{2}(t)$}

The theory presented in the previous section is based on five hypotheses: (G1) $E(k, t)=K(t) L(t) f\left(k L, R_{0}\right)$ and $(\mathrm{G} 2) \Pi(k, t)=P(t) g\left(k L, R_{0}\right)$ for the entire wavenumber range; (G3) $d L^{2} / d t$ independent of viscosity; (G4) $d c_{1} / d R_{0}=0$; and (G5) $d\left(c_{2} g\right) / d R_{0}=0$. In this section we discuss a necessary but not sufficient condition for (G1).

This condition is that $\epsilon^{>}(2 \pi / L, t)$ and

$$
\epsilon^{<}(2 \pi / L, t) \equiv 2 \nu \int_{0}^{2 \pi / L} k^{2} E(k, t) d k,
$$

and therefore also $\epsilon(t)$, vary in time in the same way. This is implied by (G1) but the inverse implication does not hold.

Given that $\int_{0}^{2 \pi / L} k^{2} E(k) d k$ must scale as $K / L^{2}$ and defining $\Phi \equiv \epsilon^{<}(2 \pi / L, t) / \epsilon(t)$ it follows directly from

$$
\epsilon(t)=\epsilon^{>}(2 \pi / L, t)+\epsilon^{<}(2 \pi / L, t)
$$

that

$$
\epsilon(t)=\frac{C \nu}{\Phi} \frac{K}{L^{2}}
$$

where $C$ is given by $\epsilon^{<}(2 \pi / L, t)=C \nu K / L^{2}$.

Time-independence of $\Phi$ (the necessary but not sufficient condition implied by G1) is therefore equivalent to time-independence of $\epsilon L^{2} / K$, i.e. time-independence of $C_{\epsilon} R_{\lambda}$.

In the presence of large-scale coherent structures in the turbulence, the time-independence of $\Phi$, equivalently of $\epsilon^{<}(2 \pi / L, t) / \epsilon^{>}(2 \pi / L, t)$, could be interpreted as follows: if the large-scale coherent vortex was to have too little dissipation then it would last comparatively long and have the time to organise the random small-scale turbulence with the result to decrease $\epsilon^{>}(2 \pi / L, t)$ and bring the ratio $\epsilon^{<}(2 \pi / L, t) / \epsilon^{>}(2 \pi / L, t)$ back up; but if the large-scale coherent vortex was to have too much dissipation, i.e. if $\epsilon^{<}(2 \pi / L, t)$ was too high, then its energy would decay too fast and its strain rates would also decay too fast given that they directly depend on the coherent energy. This would bring $\epsilon^{<}(2 \pi / L, t)$, and therefore the ratio $\epsilon^{<}(2 \pi / L, t) / \epsilon^{>}(2 \pi / L, t)$, back down. As a result, one might expect $\epsilon^{<}(2 \pi / L, t) / \epsilon^{>}(2 \pi / L, t)$, and therefore $\Phi$, to be about constant in time in the presence of large-scale coherent structures. This picture is consistent with the existence of a hierarchy of vortices of various sizes sustained by the large-scale coherent structures [7]. In this picture, vortices at each level of the hierarchy are sustained by the larger-scale strain of vortices one level larger than them and the ratio of strain rates at different consecutive length-scale levels is the same at all levels. This ratio is kept unchanged during decay causing $\epsilon^{<}(2 \pi / L, t)$ which is determined by the strain of the large-scale coherent vortices to decay together with $\epsilon^{>}(2 \pi / L, t)$ which is determined by the smaller scale strains.

From (7), this coherent structure-related timeindependence of $\Phi$ implies time-independence of $\epsilon L^{2} / K$, i.e. of $C_{\epsilon} R_{\lambda}$. The presence of such structures in the time range $t \leq t_{c}$ is evidenced by the spectral spikes at $k L \approx 2$ in Fig. 6 where we plot the three-dimensional energy spectrum of the present DNS turbulence in the time-range $t_{g} \leq t \leq t_{c}$. These spectral spikes are absent 


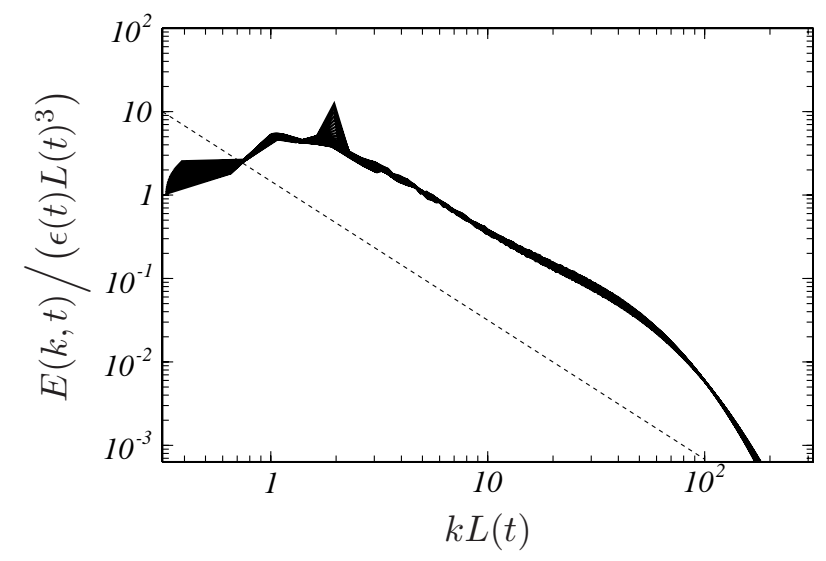

FIG. 6. Normalised energy spectra at times $t$ in the range $t_{g} \leq t \leq t_{c}$ for simulation $N=2048$. The normalisation follows $E(k, t)=\frac{\epsilon L^{3}}{\sqrt{K_{0}} L_{0}} f(k L)$ as explained in section VI. The dotted line is proportional to $(k L)^{-5 / 3}$.

from the spectra at times $t>t_{c}$ (not shown for brevity), and it is indeed at times $t<t_{c}$ that our data show some evidence of $C_{\epsilon} R_{\lambda}$ being constant in time whereas $C_{\epsilon} R_{\lambda}$ is definitely not constant at times $t>t_{c}$.

The point we are making in this section is that, by using (7), we can transform the statement that $C_{\epsilon} R_{\lambda}$ is constant in time into a different but equivalent statement which can be more easily interpreted physically. This new statement is that $\Phi=\epsilon^{<}(2 \pi / L, t) / \epsilon$ is constant in time and we have provided an example of how it might be interpreted physically two paragraphs above. Proving or disproving this interpretation is a major task and we must leave it for future works, but the task is now defined. However, as explained in the following paragraph, equation (7) can also be used to shed some more light on how well $C_{\epsilon} R_{\lambda} \approx$ Const fits our data particularly if we complete the Reynolds number dependence and note that $C_{\epsilon} R_{\lambda} \sim \sqrt{R_{0}}$ is equivalent to $\Phi \sim R_{0}^{-1}$.

In Fig. 7 we plot $\overline{R_{0} \epsilon^{<}(2 \pi / L, t) / \epsilon(t)}$ versus $\widehat{t}$ for our five DNS ensembles. This plot is in fact nearly identical to $\bar{R}_{0} \bar{\epsilon}^{<}(2 \pi / \bar{L}, t) / \bar{\epsilon}(t)$ versus $\widehat{t}$. Fig. 7 does give some support to the time-independence of $\overline{\epsilon^{<}(2 \pi / L, t) / \epsilon(t)}$ (and of $\overline{\epsilon^{<}}(2 \pi / \bar{L}, t) / \bar{\epsilon}(t)$ also given that it is about the same) in the time range $t_{g}<t<t_{c}$, particularly for our simulation ensemble $N=1024$. This simulation ensemble has the second highest Reynolds numbers (see Fig. 2 ) and covers $N_{R}=10$ realisations whereas we have only one realisation for our $N=2048$ simulation which has the highest Reynolds numbers. Given the sensitivity to initial conditions reported in section III, some single realisations of the $N=1024$ ensemble do not return values of $R_{0} \epsilon^{<}(2 \pi / L, t) / \epsilon(t)$ which are as constant in time as in Fig. 7, which means that we cannot expect the $N=2048$ curve in this figure to be statistically representative. For our DNS to agree with the constancy in time of $\overline{\epsilon^{<}(2 \pi / L, t) / \epsilon(t)}$ in the range $t_{g}<t<t_{c}$ we

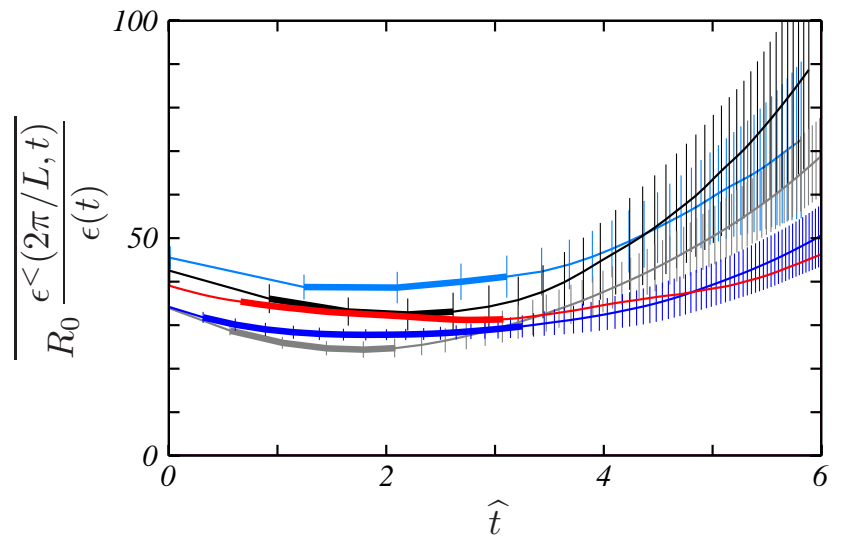

FIG. 7. Plot of $\overline{R_{0} \epsilon^{<}(2 \pi / L, t) / \epsilon}$ versus $\widehat{t}$. See Fig. 2 for the colour coding corresponding to different Reynolds numbers. The thick part of the lines marks the range $t_{g} \leq t \leq t_{c}$. The vertical bars on the lines indicate the magnitude of the standard deviation in the statistical ensemble.

need a combination of high Reynolds numbers and sufficient number of realisations. Note that the values of $R_{\lambda}$ are all below 100 at $t \geq t_{g}$ for the simulation ensembles $N=128,256,512$ (see Fig. 2) and that the best DNS agreement with $C_{\epsilon} R_{\lambda} \sim \sqrt{R_{0}}$ was obtained by [8] for their DNS which could reach values of $R_{\lambda}$ equal to or larger than about 200. The various wind tunnel experiments where this new dissipation scaling has been observed (see $[4,15,25])$ also reached such relativey high $R_{\lambda}$ values. This may explain the imperfect collapse in Fig. 4 and more clearly in the equivalent Fig. 7. It must be stated, however, that multiplying $\epsilon^{<}(2 \pi / L, t) / \epsilon(t)$ by $R_{0}$ to plot $\overline{R_{0} \epsilon^{<}(2 \pi / L, t) / \epsilon(t)}$ instead of $\overline{\epsilon^{<}(2 \pi / L, t) / \epsilon(t)}$ in Fig. 7 has definitely helped to bring the curves close to each other. Note, finally, how these curves diverge and grow in the range $t>t_{c}$, in agreement with our observation that $\overline{C_{\epsilon}}$ changes behaviours when the critical time $t_{c}$ is crossed. Equation (7) shows that the slow varying, perhaps close to constant, behaviour of $\overline{C_{\epsilon}}$ at times $t>t_{c}$ (see Fig. 2) agrees with the growth of $\overline{\epsilon^{<}(2 \pi / L, t) / \epsilon(t)}$ at these times. These are also the times when there is no evidence of large-scale coherent structures in the energy spectrum in agreement with our interpretation of the constancy in time of $\overline{\epsilon^{<}(2 \pi / L, t) / \epsilon(t)}$ in the range $t_{g}<t<t_{c}$ which relies on the presence of large-scale coherent structures as is indeed clear in the spectrum.

Our DNS of decaying turbulence cannot reach Reynolds numbers that are high enough to clearly demonstrate the independence of the turbulence dissipation rate on viscosity even though our two simulation ensembles with the highest Reynolds numbers seem to return curves close to each other in Fig. 3. In the following section we advance a few hypotheses which can help us make some progress beyond (7) and also obtain a scaling for the energy spectrum. 


\section{FROM (7) TO $\epsilon(t) \sim \sqrt{K_{0}} L_{0} K(t) / L^{2}(t)$ AND SPECTRAL SCALING}

In dealing with the time range $t_{g}<t<t_{c}$, we replace hypothesis (G1) with a set of two hypotheses (H1) and (H2) which, even when combined together, are weaker than (G1): (H1) $\Phi$ is independent of time and (H2) $E(k, t)=A(t) f\left(k L, R_{0}\right)$ for $k L \gtrsim 1$ where $f$ is a dimensionless function. As we have seen in the previous section (see paragraph containing (7)), (H1) immediately implies that the time dependencies of $\epsilon(t)$ and $K(t) / L^{2}(t)$ are the same, a result also obtained in the theory described in section IV as a direct consequence of (G1) which is far stronger than $(\mathrm{H} 1)$.

We drop hypothesis (G2). Unlike (G1) and (G2), hypotheses (H1) and (H2) allow spectral dynamics at wavenumbers above and below $L^{-1}$ to be different, as observed in our DNS. Note that (H2) is only for $k L \gtrsim 1$, not for the entire wavenumber range as is assumption (G1).

To show that $\epsilon$ is independent of viscosity and therefore $\epsilon \sim \sqrt{K_{0}} L_{0} K / L^{2}$, the theory in section IV makes use of hypotheses (G2), (G3) and (G4). Here we drop all three of them and replace them with a single hypothesis (H3) which we introduce at the appropriate place below. We also drop (G5) which was needed in section IV to derive a relation between $\Pi(k, t)$ and $\epsilon$.

From $(\mathrm{H} 2),(3)$, and $(\mathrm{H} 1)$ in the form $\epsilon^{>}(2 \pi / L, t)=$ $\epsilon(t)\left(1-\Phi\left(R_{0}\right)\right)$ it follows that

$$
A(t) \int_{2 \pi}^{\infty} \kappa^{2} f\left(\kappa, R_{0}\right) d \kappa=\frac{\epsilon}{2 \nu} L^{3}\left(1-\Phi\left(R_{0}\right)\right) .
$$

It then follows that the time dependence of $A(t)$ must be the same as the time dependence of $\epsilon(t) L^{3}(t)$ and more specifically that

$$
A(t)=\frac{\epsilon L^{3}}{\sqrt{K_{0}} L_{0}} J^{-1}
$$

where

$$
J \equiv R_{0}^{-1}\left(1-\Phi\left(R_{0}\right)\right)^{-1} I_{2}(2 \pi)
$$

with

$$
I_{2}\left(x, R_{0}\right) \equiv \int_{x}^{\infty} \kappa^{2} f\left(\kappa, R_{0}\right) d \kappa .
$$

We therefore get

$$
E(k, t)=\frac{\epsilon L^{3}}{\sqrt{K_{0}} L_{0}} J^{-1} f\left(k L, R_{0}\right)
$$

where $J$ depends only on $R_{0}$. Fig. 6 shows that our DNS offers acceptable support for this scaling, hence for (H2), at wavenumbers $k L>3$ and times $t_{g}<t<t_{c}$. The spectra are in principle hardest to collapse in this time range because this is the time range where $R_{\lambda}$ varies the most. Note that the spectra at wavenumbers $k L \leq 2$ evolve in a way that cannot be described by the self-similar form (8) and that the absence of such self-similarity is linked to the presence of large-scale coherent structures as evidenced by the evolving spike at $k L \approx 2$ in the spectrum (Fig. 6). Hypothesis (G1) is too strong as it imposes single-scale self-similarity throughout the spectrum and can therefore not account for the different evolution of large-scale coherent structures. On the contrary, hypotheses (H1) and (H2) allow spectral dynamics at wavenumbers below and above $2 L^{-1}$ to be different.

Now note that $\int_{0}^{2 \pi / L} E(k, t) d k$ must scale as $K$. Given that $K(t)=\int_{0}^{\infty} E(k, t) d k$, it then follows that $K(t) \sim$ $\int_{2 \pi / L}^{\infty} E(k, t) d k$ and using (8) yields $I_{0}(2 \pi) J^{-1} \epsilon \sim$ $\sqrt{K_{0}} L_{0} K / L^{2}$ where $I_{0}\left(x, R_{0}\right) \equiv \int_{x}^{\infty} d \kappa f\left(\kappa, R_{0}\right)$. It is here that the extra hypothesis (H3) is needed. This hypothesis states that the ratio $I_{0}\left(2 \pi, R_{0}\right) / J\left(R_{0}\right)$ does not depend on $R_{0}$ in the limit where $R_{0} \rightarrow \infty$. Hence

$$
\epsilon(t) \sim \sqrt{K_{0}} L_{0} K(t) / L^{2}(t)
$$

One can work out from (5) that (G3) and (G4) play the same role as (H3) in ensuring that the turbulence dissipation is independent of viscosity and that (H3) is a little weaker. In fact, hypothesis (H3) replaces hypotheses (G2), (G3) and (G4) but is admitedly as ad hoc as (G4). However, (H3) does have the merit of being expressed in terms of integral quantities involving only the energy spectral shape $f$ and its dependence on Reynolds number. One can therefore try different functions $f$ and see whether they are consistent with (H3) and thereby (9). For example a function $f$ which is proportional to $(k L)^{-5 / 3}$ and independent of $R_{0}$ for $1<k L \leq L / \eta \equiv R_{0}^{4 / 3}$ and has a sharp fall off with $k L$ at $k \eta \leq 1$ (as in Fig. 6) satisfies (H3), and also (H2). One cannot make such a statement for (G4) which is therefore more opaque than (H3). Equation (4) makes it clear that an asssessment of (G4), i.e. of $d c_{1} / d R_{0}=0$, would involve both functions $f$ and $g$ as well as the other dimensionless coefficients $c_{2}$ and $c_{3}$ of the theory in section IV.

In summary, hypothesis (G1) has been replaced by the weaker set of hypotheses (H1) and (H2) which yielded the spectral scaling (8), the most important result of this section, and hypotheses (G3) and (G4) have been replaced by the weaker hypothesis (H3). (G2) and (G5) which concern the interscale energy flux have been dropped. In the next section we derive expressions for the energy flux in the time range $t_{g}<t<t_{c}$ with no further hypotheses and no use of (H3). 


\section{THE ENERGY FLUX $\Pi(k, t)$ AT TIMES SHORTER THAN $t_{c}$}

We derive a formula for $\Pi(k, t)$ for $k L \gtrsim 1$ by using (1), (2) and (8):

$$
\begin{aligned}
\sqrt{K_{0}} L_{0} \Pi(k, t)= & \sqrt{K_{0}} L_{0} \epsilon^{>}(k, t)-\frac{\epsilon}{2} \frac{d L^{2}}{d t} J^{-1} k L f\left(k L, R_{0}\right) \\
& +\frac{d}{d t}\left(\epsilon L^{2}\right) J^{-1} I_{0}\left(k L, R_{0}\right)
\end{aligned}
$$

for $k L \gtrsim 1$. This expression implies that, at any given time $t$ in the range $t_{g}<t<t_{c}, \Pi(k, t)-\epsilon^{>}(k, t)$ is proportional to $(k L)^{1-p}$ if $f \propto(k L)^{-p}$. Fig. 8, where we plot $\left[\Pi(k, t)-\epsilon^{>}(k, t)\right] /\left[\Pi(2 \pi / L, t)-\epsilon^{>}(2 \pi / L, t)\right]$ versus $k L$, shows that this is not too far from being the case with $p$ close to $5 / 3$. The Kolmogorov theory which predicts $p=5 / 3$ (for steady turbulence) does not predict such a dependence of $\Pi(k, t)$ on wavenumber.

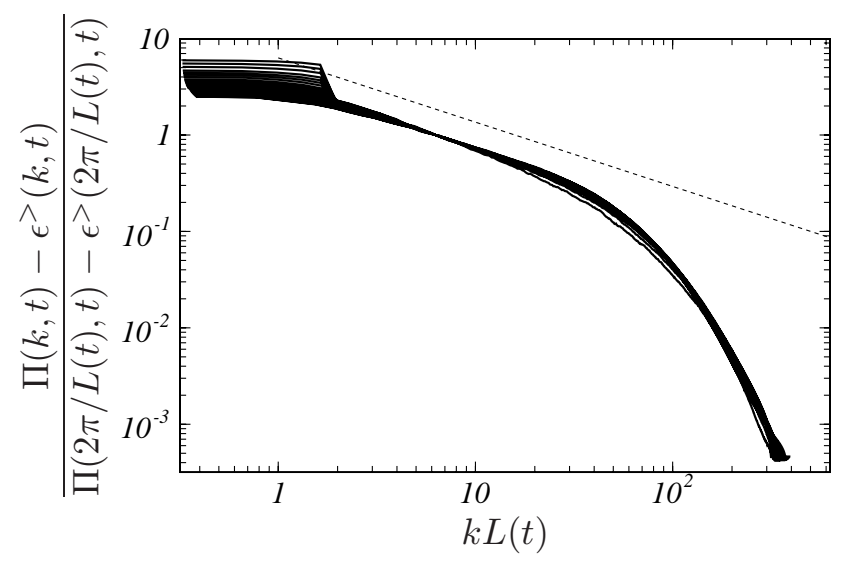

FIG. 8. Plot of $\left[\Pi(k, t)-\epsilon^{>}(k, t)\right] /\left[\Pi(2 \pi / L, t)-\epsilon^{>}(2 \pi / L, t)\right]$ versus $k L$ for various times in the range $t_{g}<t<t_{c}$. $N=$ 2048. The dotted line is proportional to $(k L)^{-2 / 3}$.

To show that our hypotheses (H1) and (H2) are consistent with our observation that $\Pi(k, t) / \epsilon^{>}(k, t)$ is a decreasing function of time till $t=t_{c}$ (Fig. 5) we look at the time dependence in expression (10). This expression involves the time derivative of $\epsilon$ which we evaluate by differentiating (7) with respect to time and by making use of (H1) in the form $\frac{d \Phi}{d t}=0$. Making also use of $\frac{d K}{d t}=-\epsilon$ we obtain

$$
\frac{d \epsilon}{d t}=-\frac{\epsilon^{2}}{K}-\frac{\epsilon}{L^{2}} \frac{d L^{2}}{d t} .
$$

By injecting (11) in (10) and making one more use of (7) we conclude that

$$
\begin{aligned}
& -\frac{\partial K^{>}(k, t)}{\partial t} / \epsilon^{>}(k, t) \\
& =1-\frac{\Pi(k, t)}{\epsilon^{>}(k, t)} \\
& =\frac{\epsilon(t)}{2 \epsilon^{>}(k, t)}\left[\frac{d L^{2}}{d t} \frac{k L f\left(k L, R_{0}\right)}{\sqrt{K_{0}} L_{0} J\left(R_{0}\right)}+\frac{2 C}{\Phi R_{0}} \frac{I_{0}\left(k L, R_{0}\right)}{J\left(R_{0}\right)}\right]
\end{aligned}
$$

for $k L \gtrsim 1$.

Expression (12) makes it clear that $\frac{\Pi(k, t)}{\epsilon^{>}(k, t)}$ is a decreasing function of time for a given inertial range wavenumber $k$ mainly because of the growth of $\frac{d L^{2}}{d t}$, but also, albeit to a lesser exent, because of the relatively slow growth of $\frac{\epsilon(t)}{\epsilon^{>}(k, t)}$. This demonstrates that hypotheses (H1) and (H2) which imply (12) are consistent with the increasing inertial range non-stationarity evidenced in Fig. 5 for any given inertial range wavenumber. They are also consistent with the observation (both in Fig. 5 and in [8]) that there is a tendency towards Kolmogorov stationarity at the higher wavenumber end of the inertial range. This is clearly evident if we set $f(k L) \sim(k L)^{-5 / 3}$ in the inertial range which additionaly shows that this tendency is slow because it scales as $k^{-2 / 3}$.

Expression (12) for $\frac{\Pi(\dot{k}, t)}{\epsilon^{>}(k, t)}-1$ can also be used to predict $\Pi(2 \pi / L(t), t)$ by setting $k=2 \pi / L$ and using (H1) in the form $\epsilon^{>}(2 \pi / L, t)=\epsilon(t)(1-\Phi)$ :

$$
\begin{aligned}
\frac{\Pi(2 \pi / L, t)}{\epsilon(t)}= & 1-\Phi\left(R_{0}\right)-\frac{d L^{2}}{d t} \frac{\pi f\left(2 \pi, R_{0}\right)}{\sqrt{K_{0}} L_{0} J\left(R_{0}\right)} \\
& -\sqrt{\frac{3}{2}} \frac{C}{\Phi R_{0}} \frac{I_{0}\left(2 \pi, R_{0}\right)}{J\left(R_{0}\right)}
\end{aligned}
$$

Clearly, hypotheses (H1) and (H2) imply that $\Pi(2 \pi / L, t)$ and $\epsilon(t)$ do not have the same time dependence at times $t<t_{c}$. In Fig. 9 we plot both $\overline{\Pi(2 \pi / L, t) / \epsilon}$ and $\overline{\Pi(2 \pi / L, t) /\left(u^{\prime 3} / L\right)}$ versus $\widehat{t}$ (which turn out to be effectively identical with the respective plots of $\bar{\Pi}(2 \pi / \bar{L}, t) / \bar{\epsilon}$ and $\bar{\Pi}(2 \pi / \bar{L}, t) /\left({\overline{u^{\prime}}}^{3} / \bar{L}\right)$ versus $\left.\widehat{t}\right)$. The decrease of $\overline{\Pi(2 \pi / L, t) / \epsilon}$ is in agreement with the increase of $\frac{d \bar{L}^{2}}{d t}$. The constancy in time of $\overline{\Pi(2 \pi / L, t) / \epsilon}$ and $\overline{\Pi(2 \pi / L, t) /\left(u^{\prime 3} / L\right)}$ for sufficiently high $\overline{R_{0}}$ in the range $t>t_{c}$ cannot be accounted for by (13) as $\frac{d L^{2}}{d t}$ decreases with time in that range. In the next section we briefly discuss the time range $t>t_{c}$.

\section{TIMES LONGER THAN $t_{c}$}

From Fig. 9, equation (13) and the dissipation law $\epsilon(t) \sim K(t) / L^{2}(t)$ we deduce that $\Pi(2 \pi / L, t)$ does not scale as $u^{\prime 3} / L$ in the time range $t<t_{c}$. Such a scaling is not supported by either hypotheses (H1) and (H2) or by our DNS results. However, Fig. 9 also shows that $\Pi(2 \pi / L, t)$ does scale as $u^{\prime 3} / L$ in the time range $t>t_{c}$ 

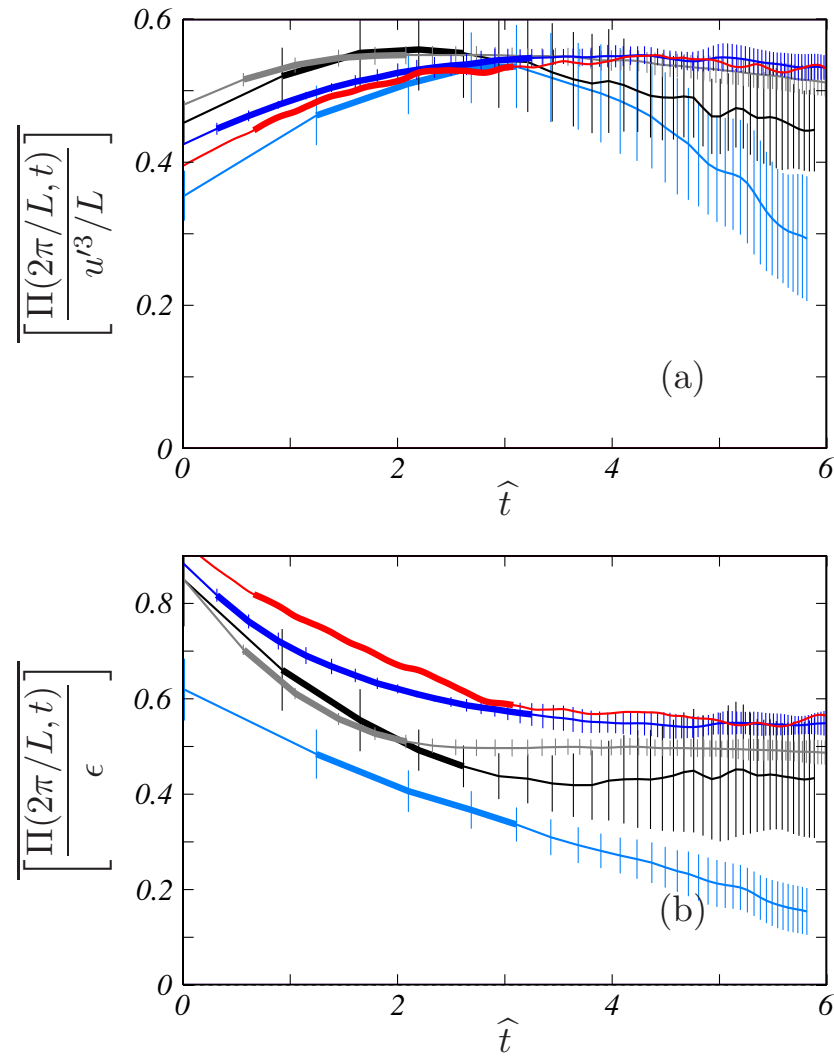

FIG. 9. (a) $\overline{\Pi(2 \pi / L, t) /\left(u^{\prime 3} / L\right)}$ versus $\widehat{t}$. (b) $\overline{\Pi(2 \pi / L, t) / \epsilon}$ versus $\widehat{t}$. See Fig. 2 for the colour coding corresponding to different Reynolds numbers. The thick part of the lines marks the range $t_{g} \leq t \leq t_{c}$. The vertical bars on the lines indicate the magnitude of the standard deviation in the statistical ensemble.

provided $R_{0}$ is large enough. Given that $\Pi(2 \pi / L, t)$ and $\epsilon(t)$ vary in time in the same way in this far time range as seen in Fig. 9(b), it follows that this time range is such that $\epsilon(t) \sim u^{\prime 3} / L(t)$. Fig. 3(b) supports this conclusion.

This conclusion might be a little surprising because the far time range is the lower Reynolds number range for each one of our simulation ensembles and because the turbulence is far from Kolmogorov stationarity in this range too (see Fig. 5). It would therefore appear that the decaying turbulence in the far time range $t>t_{c}$ is close to a state of balanced non-equilibrium (in fact not perfectly so as Fig. 5 suggests) which is a state where all three terms in (1) remain approximately proportional to each other during decay for $k \sim 1 / L(t)$ (see also [26]). This is the only way that a turbulence can be out of Kolmogorov stationarity while at the same time approximately respecting $\Pi(2 \pi / L, t) \sim \epsilon(t)$ and the Taylor-Kolmogorov scaling $\epsilon \sim u^{\prime 3} / L[11,19]$. Indeed, the presence and influence of the large-scale coherent vortices having subsided in the long time regime $t>t_{c}$, the memory of initial conditions at $t=t_{0}$ has also subsided meaning that $\Pi(2 \pi / L(t), t)$ must scale as $u^{\prime 3}(t) / L(t)$ and therefore

$$
\epsilon(t) \approx \epsilon^{>}(2 \pi / L(t), t) \sim u^{\prime 3}(t) / L(t)
$$

by virtue of the approximate balanced non-equilibrium. This is very different from Kolmogorov stationarity which implies $\Pi(2 \pi / L(t), t) \approx \epsilon(t)$ instead of $\Pi(2 \pi / L(t), t) \sim$ $\epsilon(t)$. Indeed, $\Pi(2 \pi / L(t), t) \approx 0.55 \epsilon(t)$ for the highest values of $R_{0}$ in Fig. 9(b).

One may ask whether the time $t_{c}$ results from or is at least partly determined by the finite size of the periodic DNS box. Even though the integral scale is less than a tenth of the box size from $\widehat{t}=0$ to $\widehat{t}=4$ for our three simulation ensembles with the highest Reynolds numbers and even though previous studies such as [21] have determined that this is enough for a fair determination of the integral length-scale, we cannot rule out such a finite size effect. Nevertheless, the classical $\Pi(2 \pi / L(t), t) \sim \epsilon(t) \sim u^{\prime 3} / L(t)$ are present at times $t>t_{c}$, i.e. $\widehat{t}>3.2$, in the three DNS ensembles with the highest $R_{0}$. If the finite size box is responsible for the existence or even just the value of $t_{c}$, one would then have to ask if it is also, at least partly, responsible for these classical scalings and the related non-equilibrium balance. DNS runs with an even higher box size to $\bar{L}$ ratio and same Reynolds number and small-scale resolution would be needed to answer this question and this is not currently feasible. We recall, however, that dissipation scalings such as the ones obtained in the present DNS have also been obtained in wind tunnels [25], the classical scaling appearing downstream of the scaling (9) as in our DNS, for various ratios of the wind tunnel size to the integral scale including significantly larger than 10 .

\section{CONCLUDING DISCUSSION}

It can be expected that the most common nonlinear turbulence cascades in nature and engineering are unsteady energy cascades. We have identifed two different types of unsteady energy cascade for incompressible three-dimensional turbulence, each one with a different turbulence dissipation scaling associated to it. And we have also shown that, during decay, the turbulence transits quite abruptly from one unsteady energy cascade to the other. Both these cascades are fundamentally different from Kolmogorov's statistically stationary cascade.

It has proved to be computationally extremely demanding to obtain reliable cascade-related information from DNS of decaying periodic turbulence because of the need for high enough Reynolds numbers combined with large numbers of realisations and long integration times. Our DNS have nevertheless unambiguously demonstrated that a critical time $t_{c}$ exists when there is a simultaneous qualitative change in (i) the instantaneous scalings of $\epsilon(t)$, (ii) the ratio of the interscale energy flux to the high-pass turbulence dissipation which quantifies the non-stationarity of the small-scale turbulence, (iii) the rate of change of the integral length-scale and (iv) the shape of the energy spectrum at length-scales comparable to $L$. 
(i) The turbulence dissipation rate coefficient changes from a scaling comparable to $C_{\epsilon} \sim \sqrt{R_{0}} / R e_{\lambda}$ which was also found in various other wind tunnel and DNS turbulent flows $[4,8,9,15,25]$ to $C_{\epsilon} \approx$ Const at times $t>t_{c}$ and high enough values of $R_{0}$.

(ii) The ratio of the interscale energy flux to the highpass filtered turbulence dissipation starts out as a decreasing function of time till $t=t_{c}$ when it stops decreasing and proceeds to very slowly increase. This behaviour is observed for all inertial range wavenumbers. It means that the cascade becomes increasingly non-stationary till the critical time $t_{c}$ when the cascade starts to slowly decrease its degree of non-stationarity. However the cascade remains highly non-stationary throughout our simulations, even when $C_{\epsilon} \approx$ Const at times $t>t_{c}$. We introduce the concept of balanced non-equilibrium to explain this long time behaviour but warn that it should not be expected to be perfectly present in general as $C_{\epsilon} \approx$ Const is also often not perfectly present, for example in far-downstream grid-generated turbulence (see Fig. 4 in [22]).

(iii) $\frac{d L^{2}}{d t}$ changes from an increasing function of time at times $t<t_{c}$ to a decreasing function at times $t>t_{c}$.

(iv) At times $t<t_{c}$ there is a clear peak in the energy spectrum which suggests the presence of large-scale coherent structures at length-scales comparable to $L$. This spectral peak disappears after the critical time $t_{c}$.

One is naturally led to an interpretation of our results in terms of effective predominance of large-scale coherent structures at times $t<t_{c}$ and absence if not just weak presence of such structures at times $t>t_{c}$. This interpretation, which we relate to a physically meaningful way of stating the new dissipation scaling in section $\mathrm{V}$, is also supported by previously published data. For example, the new dissipation scaling has turned out to be better defined in regular grid than in fractal grid turbulence (see [24]) and [12] have shown that the vortex shedding signature is much stronger in regular grid than in fractal grid turbulence. The new mean wake scalings which result from the new dissipation scaling are also much better defined for the low than for the high iteration fractal plates used by [14] as wake generators (see their table II), and [13] have shown that the vortex shedding signature is much stronger in low than in high iteration fractal plate wakes (see their Fig. 13b). In fact, the wake scalings seem to tend towards the classical ones which follow from $C_{\epsilon}=$ Const as the vortex shedding signature decreases. Finally, [12] have recently shown for grid-generated turbulence that the integral scale increases faster in the presence of large-scale coherent structures than in the absence of such vortex shedding structures.

To account for the effects of the large-scale coherent structures on the turbulence cascade and dissipation at times $t<t_{c}$, we have therefore advanced two hypotheses, (H1) and (H2), in section VI. These hypotheses lead to the scaling (8) of the energy spectrum which is fairly well supported by our DNS and to equation (12) for the interscale energy flux which agrees qualitatively with the increasingly non-stationary nature of the turbulence cascade at times $t<t_{c}$. Our DNS show that, at times $t<t_{c}$, the turbulence enegy spectrum has a well-defined $k^{-5 / 3}$ range at high Reynolds numbers. This spectrum is nevertheless very different from Kolmogorov's $E(k) \sim \epsilon^{2 / 3} k^{-5 / 3}$ and scales as $E(k) \sim \frac{\epsilon L^{3}}{\sqrt{K_{0}} L_{0}}(k L)^{-5 / 3} \sim K L(k L)^{-5 / 3}$ (using $\epsilon \sim$ $\left.\sqrt{K_{0}} L_{0} K / L^{2}\right)$ as originally concluded by [18] from their wind tunnel experiments.

Finally it is worth mentioning that (H1) and (H2) also lead to equation (11) which is reminiscent of the $K-\epsilon$ equation for homogeneous turbulence. It is conceivable that one might therefore consider future possibilities of improved one-point turbulence modelling for unsteady turbulence in the presence of large-scale coherent structures.

SG is supported by JSPS Grants-in-Aid for Scientific Research 25249014, 26630054, 16H04268 and JCV is supported by an ERC Advanced Grant.

[1] G.I. Barenblatt and A.A. Gavrilov. On the theory of selfsimilar degeneracy of homogeneous isotropic turbulence. Sov. Phys. JETP, 38(2):399-402, 1974.

[2] W. Bos and R. Rubinstein. Dissipation in unsteady turbulence. 2016.

[3] J.I. Cardesa, A. Vela-Martin, S. Dong, and J. Jimenez. The temporal evolution of the energy flux across scales in homogeneous turbulence. Physics of Fluids (1994present), 27(11):111702, 2015.

[4] T. Dairay, M. Obligado, and J.C. Vassilicos. Nonequilibrium scaling laws in axisymmetric turbulent wakes. Journal of Fluid Mechanics, 781:166-195, 2015.

[5] U. Frisch. Turbulence. Cambridge university press, 1995.

[6] W.K. George. The decay of homogeneous turbulence. Physics of Fluids A, 4(7):1492-1509, 1992.

[7] S. Goto and G. Kawahara. Generation mechanism of hierarchy of coherent vortices in turbulence sustained by steady force. Proc. 14th European Turbulence Conference, 2016.

[8] S. Goto and J.C. Vassilicos. Energy dissipation and flux laws for unsteady turbulence. Physics Letters A, 379:1144-1148, 2015.

[9] S. Goto and J.C. Vassilicos. Local equilibrium hypothesis and taylor's dissipation law. Fluid Dynamics Research, 48(2):021402, 2016.

[10] R.J. Hearst and P. Lavoie. Decay of turbulence generated by a square-fractal-element grid. Journal of Fluid Mechanics, 741:567-584, 2014.

[11] A.N. Kolmogorov. On degeneration (decay) of isotropic turbulence in an incompressible viscous fluids. Dokl. Akad. Nauk. SSSR, 31:538-540, 1941.

[12] G. Melina, P.J.K. Bruce, and J.C. Vassilicos. Vortex shedding effects in grid-generated turbulence. Physical Review Fluids, 1:044402, 2016.

[13] J. Nedić, B. Ganapathisubramani, and J.C. Vassilicos. Drag and near wake characteristics of flat plates normal to the flow with fractal edge geometries. Fluid Dynamics 
Research, 45(6):061406, 2013.

[14] J. Nedić, J.C. Vassilicos, and B. Ganapathisubramani. Axisymmetric turbulent wakes with new nonequilibrium similarity scalings. Physical Review Letters, 111(14):144503, 2013.

[15] M. Obligado, T. Dairay, and J.C. Vassilicos. Nonequilibrium scalings of turbulent wakes. Physical Review Fluids, 1:044409, 2016.

[16] L.I. Sedov. Similarity and dimensional methods in mechanics. Academic Press, New York, 1959.

[17] L.I. Sedov. Decay of isotropic turbulent motions of an incompressible fluid. Dokl. Akad. Nauk. SSSR, 1974.

[18] R.E. Seoud and J.C. Vassilicos. Dissipation and decay of fractal-generated turbulence. Physics of Fluids (1994present), 19(10):105108, 2007.

[19] G.I. Taylor. Statistical theory of turbulence. Proc. R. Soc. Lond. A, 151:421-444, 1935.

[20] H. Tennekes and J. L. Lumley. A first course in turbulence. MIT press, 1972.

[21] B. Thornber. Impact of domain size and statistical errors in simulations of homogeneous decaying turbulence and the richtmyer-meshkov instability. Physics of Fluids (1994-present), 28(4):045106, 2016.
[22] P. Valente and J.C. Vassilicos. Dependence of decaying homogeneous isotropic turbulence on inflow conditions. Physics Letters A, 376:510-514, 2012.

[23] P. Valente and J.C. Vassilicos. Universal dissipation scaling for non-equilibrium turbulence. Physical Review Letters, 108(12):214503, 2012.

[24] P. Valente and J.C. Vassilicos. The non-equilibrium region of grid-generated decaying turbulence. Journal of Fluid Mechanics, 744:5-37, 2014.

[25] J.C. Vassilicos. Dissipation in turbulent flows. Ann. Rev. Fluid Mech., 47(1), 2015.

[26] S.L. Woodruff and R. Rubinstein. Multiple-scale perturbation analysis of slowly evolving turbulence. Journal of Fluid Mechanics, 565:95-103, 2006.

[27] P.K. Yeung, D.A. Donzis, and K.R. Sreenivasan. Dissipation, enstrophy and pressure statistics in turbulence simulations at high reynolds numbers. J. Fluid Mech., 700:5-15, 2012.

[28] A. Yoshizawa. Nonequilibrium effect of the turbulentenergy-production process on the inertial-range energy spectrum. Physical Review E, 49(5):4065, 1994. 